\title{
Clinical Impact of Perioperative Oral Nutritional Treatment for Body Composition Changes in Gastrointestinal Cancer Treatment
}

\author{
TORU AOYAMA ${ }^{1,2^{*}}$, MASATO NAKAZONO $^{1,2^{*}}$, SHINNOSUKE NAGASAWA $^{1,2}$ and KENKI SEGAMI ${ }^{1,2}$ \\ ${ }^{1}$ Department of Surgery, Yokohama City University, Yokohama, Japan; \\ ${ }^{2}$ Department of Gastrointestinal Surgery, Kanagawa Cancer Center, Yokohama, Japan
}

\begin{abstract}
The standard treatment for gastrointestinal cancer is surgical resection and perioperative adjuvant treatment. Multidisciplinary treatment for gastrointestinal cancer leads to body composition changes. Body composition changes, such as skeletal muscle loss and body weight loss, during multidisciplinary treatment result in poor physical activity, severe toxicity of chemotherapy and/or radiation therapy, and poor oncological outcomes. Therefore, the hypothesis is that minimization of body composition changes during multidisciplinary treatment in gastrointestinal cancer patients, the continuation of postoperative adjuvant treatment in these patients might improve, thereby improving the oncological outcomes. Given this hypothesis, recent studies have focused on introducing perioperative oral nutritional treatment for gastrointestinal cancer patients. Thus far, oral nutritional treatment has proven promising and showed some clinical benefits for gastrointestinal cancer patients during the perioperative period. However, whether or not oral nutritional treatment has clinical benefits on the long-term oncological outcomes in gastrointestinal cancer remains unclear. To optimize oral nutritional treatment for gastrointestinal cancer patients, it is necessary to clarify the benefits of oral nutritional treatment on the long-term oncological outcomes in gastric cancer patients and establish the optimal approach to oral nutritional treatment.
\end{abstract}

This article is freely accessible online.

*These Authors contributed equally to this study.

Correspondence to: Toru Aoyama, Department of Surgery, Yokohama City University, 3-9 Fukuura, Kanazawa-ku, Yokohama 236-0004, Japan. Tel: +81 457872800, e-mail: t-aoyama@lilac.plala.or.jp

Key Words: Oral nutritional treatment, gastric cancer, esophageal cancer, review.
An estimated 14.1 million new cancer cases and 8.2 million cancer deaths occurred in 2012 worldwide (1). According to various treatment guidelines, the standard treatment for gastrointestinal cancer is surgical resection and perioperative adjuvant treatment (2-6). Multidisciplinary treatment for gastrointestinal cancer leads to body composition changes, such as body weight loss and skeletal muscle loss. Especially, gastric cancer patients and esophageal cancer patients develop severe body composition changes during multidisciplinary treatment $(7,8)$. Recent studies demonstrated that body composition changes affect both short- and long-term oncological outcomes. Also, perioperative body weight loss and skeletal muscle loss affect the toxicity of adjuvant treatment and continuation of therapy, and also the recurrence pattern and survival (9-12). To improve both the short- and long-term oncological outcomes in gastrointestinal cancer, it is necessary to minimize perioperative body composition changes. Considering these, recent studies have focused on introducing perioperative oral nutritional treatment for gastrointestinal cancer patients.

We herein review the background, current status, and future perspectives of perioperative oral nutritional treatment for body composition changes in gastrointestinal cancer patients.

\section{Clinical Impact of Perioperative Body Composition Changes in Gastrointestinal Cancer Outcomes}

In gastric cancer, we have previously evaluated the clinical impact of postoperative body weight loss (BWL) on both the short- and long-term oncological outcomes. We compared the rate of adjuvant chemotherapy continuation between the $\mathrm{BWL}<15 \%$ and $\mathrm{BWL} \geq 15 \%$ groups in 103 locally advanced gastric cancer patients (13). We found that the 6-month continuation rate of adjuvant chemotherapy was $36.4 \%$ in the $\mathrm{BWL} \geq 15 \%$ group but $66.4 \%$ in the $\mathrm{BWL}<15 \%$ group 
$(p=0.017)$. BWL $\geq 15 \%$ was a significant risk factor associated with the discontinuation of adjuvant chemotherapy. About half of the patients with BWL $\geq 15 \%$ were unable to continue adjuvant chemotherapy for more than two courses. In the same cohort, we did a follow-up study (median follow-up: 64.3 months) (14). We found that the 5-year overall survival (OS) was $36.4 \%$ in the BWL $\geq 15 \%$ group but $59.9 \%$ in the $\mathrm{BWL}<15 \%$ group $(p=0.004)$, and the 5-year recurrence-free survival (RFS) was $36.4 \%$ in the $\mathrm{BWL} \geq 15 \%$ group but $56.4 \%$ in the $\mathrm{BWL}<15 \%$ group $(p=0.016)$. BWL was an independent prognostic factor for gastric cancer patients.

In addition, we have previously evaluated the clinical impact of postoperative lean body mass loss (LBL) on both the shortand long-term oncological outcomes (15). We compared the rate of adjuvant chemotherapy continuation between the LBL $<5 \%$ and $\mathrm{LBL} \geq 5 \%$ groups in 58 locally advanced gastric cancer patients. We found that the 6-month continuation rate of adjuvant chemotherapy was $66.3 \%$ in the $\mathrm{LBL} \geq 5 \%$ group but $91.7 \%$ in the $\mathrm{LBL}<5 \%$ group ( $p=0.031$ ). The incidence of grade 3 toxicities was higher in the LBL $\geq 5 \%$ group than in the LBL $<5 \%$ group ( $42.9 \% v s .18 .9 \%)$. We also evaluated the clinical impact of LBL on the long-term oncological outcomes in 115 locally advanced gastric cancer patients (16). We found that the 5-year RFS was $57.8 \%$ in the LBL $\geq 5 \%$ group but $73.5 \%$ in the LBL $<5 \%$ group $(p=0.040)$. LBL was an independent prognostic factor for gastric cancer patients.

In esophageal cancer, Koterazawa et al. evaluated the clinical impact of postoperative severe weight loss after esophagectomy in 317 esophageal cancer patients who received minimally invasive surgery (17). They used the patient's body weight at 3 months after surgery to divide the patients into a severe weight loss group $(\mathrm{n}=65)$ and moderate weight loss group $(\mathrm{n}=252)$. The 5 -years OS was $54 \%$ in the severe weight loss group and $67 \%$ in the moderate weight loss group $(p=0.024)$, and the 5 -year progression-free survival (PFS) was $61 \%$ in the severe weight loss group and $71 \%$ in the moderate weight loss group $(p=0.039)$. They concluded that severe weight loss was significantly associated with a poor OS in esophageal cancer patients.

In addition, Mayanagi et al. evaluated the clinical impact of postoperative severe skeletal muscle loss after esophagectomy in 66 esophageal cancer patients who received adjuvant chemotherapy followed by surgery (18). They used the patient's body composition at four months after surgery to divide patients into groups with and without a decrease in skeletal muscle. Among 66 patients, 39 (59\%) showed a skeletal muscle decrease from baseline to 4 months after esophagectomy. The 3-years OS was $68.8 \%$, and the 3year RFS was $57.3 \%$. Skeletal muscle loss was an independent prognostic factor for both the OS [hazard ratio $(\mathrm{HR})=1.16,95 \%$ confidence interval $(\mathrm{CI})=1.03-1.31$, $p=0.015]$ and $\mathrm{RFS}(\mathrm{HR}=1.11,95 \% \mathrm{CI}=1.01-1.24, p=0.048)$.
Given these previous findings, perioperative body composition changes appear to affect both the short- and long-term oncological outcomes in gastrointestinal cancer patients.

\section{Type of Perioperative Oral Nutritional Treatment for Body Composition Changes in Gastrointestinal Cancer}

Perioperative body composition changes in gastrointestinal cancer patients are mainly due to a decreased oral intake and surgical stress. There have been two approaches to managing body composition changes: immune-modulating nutrition and pharmaconutrition. Pharmaconutrition mainly focuses on improving the decreased oral intake, while immunemodulating nutrition focuses on improving the nutritional status, modulating the host immune system, and suppressing the inflammatory response to surgical stress. The most frequently recognized immune-modulating nutrients are various combinations of fish oil ( $\omega-3$ fatty acids), nucleotides, glutamine, and arginine. Both immunemodulating nutrition and pharmaconutrition aim to improve the patient's survival by minimizing perioperative body composition changes.

\section{Clinical Impact of Perioperative Oral Nutritional Treatment on Body Composition Changes in Gastric Cancer}

Four clinical trials have been performed to evaluate the influence of perioperative oral nutritional treatment on body composition changes in gastric cancer patients. Pharmaconutrition has been evaluated in three trials. Hatao et al. (19) evaluated the clinical effects of an oral nutritional supplement (ONS) on postoperative BWL in 113 gastric cancer patients who received gastrectomy (ONS, 64 patients; Control group, 49 patients). They administered the concentrated liquid diet ANOM $^{\circledR}$ (Otsuka, Tokyo, Japan) at $400 \mathrm{kcal} /$ day and continued this regimen until 12 weeks after discharge. The primary endpoint of the study was postoperative percentage weight changes at 12 weeks after surgery. They found that weight changes were $91.6 \%$ in the Control group and $91.1 \%$ in the ONS group $(p=0.26)$. A similar trend was observed in distal gastrectomy patients (Control group: $93.4 \%$ vs. ONS group: $94.1 \%, p=0.26$ ). However, in total gastrectomy patients, ONS improved the postoperative BWL (Control group: $85.6 \%$ vs. ONS group: $88.5 \%, p=0.03)$. In contrast, there were no significant differences in the skeletal muscle loss between the Control and ONS groups, regardless of the type of gastrectomy. Although the primary endpoint was not met in Hatao's study, the perioperative use of ONS might diminish the postoperative BWL in total gastrectomy patients. 
Imamura et al. evaluated whether or not an oral elemental diet (ED) prevented postoperative body weight loss in 112 gastric cancer patients who received gastrectomy (ED group, 58 patients; Control group, 54 patients) (20). They administered Elental ${ }^{\circledR}$ (Ajinomoto Pharmaceutics, Tokyo, Japan) at $300 \mathrm{kcal} /$ day and continued until 6-8 weeks after gastrectomy. The primary endpoint of the study was the postoperative percentage weight change at six to eight weeks after surgery. They found that weight changes were $93.4 \%$ in the Control group and $95.1 \%$ in the ED group ( $p=0.047$ ). There was a significant improvement in the ED group. A similar trend was observed in the total gastrectomy patients (Control group: $90.9 \%$ vs. ED group: $95.0 \%, p=0.26$ ). They also reported that the perioperative use of the ED was independently associated with body weight loss after gastrectomy (relative risk 1.797, $p=0.036$ ). They concluded that the use of an ED in the perioperative period ameliorated the postoperative weight loss after gastrectomy. In addition, they also showed that daily nutritional intervention (300 $\mathrm{kcal} /$ day ED) for 6-8 weeks reduced the percentage BWL at 1 year in patients who underwent total gastrectomy (21).

Kong et al. evaluated whether or not an ONS prevented postoperative BWL in 127 moderately to severely malnourished gastric cancer patients who underwent gastrectomy (22). They administered Ensure powder sachets (Abbott Laboratories, Lake Bluff, IL, USA) at $500 \mathrm{kcal} /$ day from 2 weeks before surgery to 4 weeks after surgery. The patients were divided into a Control group $(n=62)$ and an ONS group $(n=65)$. The primary endpoint of the study was postoperative complications, and the secondary endpoint was body weight changes. They found that the postoperative surgical complication rate was $29.2 \%$ in the ONS group and $37.1 \%$ in the Control group $(p=0.346)$. The changes in BWL were also similar between the ONS and Control groups, being 92\%-94\% at 5-6 weeks after surgery in both groups. This study suggested that the use of an ONS in the perioperative period did not markedly improve the surgical complications or BWL.

Regarding immune-modulating nutrition approaches, we have evaluated the clinical effects of oral immunonutritional on postoperative BWL and LBL in 126 gastric cancer patients who received total gastrectomy (23). We administered the oral supplementation ProSure ${ }^{\circledR}$ (Abbot Laboratories, Dublin, Ireland) at $600 \mathrm{kcal} / \mathrm{day}$ [including $2.2 \mathrm{~g}$ eicosapentaenoic acid (EPA)] from 7 days before surgery to 21 days after gastrectomy. The patients were divided into a Control group $(n=60)$ and ProSure group $(n=63)$. The primary endpoint was the percentage of BWL at one and three months after gastrectomy. We found that the weight changes at 1 month were $91.3 \%$ in the Control group and $91.5 \%$ in the ProSure group ( $p=0.818$ ), and those at 3 months were $86.5 \%$ in the Control group and $87.0 \%$ in the ProSure group $(p=0.529)$. Similar trends were observed in LBL. We found that the lean body mass changes at 1 month were $93.3 \%$ in the Control group and $93.9 \%$ in the ProSure group ( $p=0.794)$ (24), and those at 3 months were $91.4 \%$ in the Control group and $92.3 \%$ in the ProSure group $(p=0.393)$. We concluded that an immunonutritional approach based on an EPA oral diet did not reduce the BWL or LBL after total gastrectomy for gastric cancer compared with a standard diet.

\section{Clinical Impact of Perioperative Oral Nutritional Treatment for Body Composition Changes in Esophageal Cancer Treatment}

Ryan et al. have evaluated the clinical effects of EPA-enriched enteral nutrition (EN) in 53 esophageal cancer patients who received esophagectomy (EPA-enriched EN in 28 patients and standard EN in 25 patients) (25). They administered the oral supplementation ProSure ${ }^{\circledR}$ (Abbot Laboratories, Dublin, Ireland) at $600 \mathrm{kcal} / \mathrm{day}$ (including $2.2 \mathrm{~g} \mathrm{EPA}$ ) from 5 days before surgery to 21 days after esophagectomy. The patients with EPA-enriched EN maintained their fat-free mass, while those who received standard EN lost a significant amount of fatfree mass (1.9-kg reduction; $p=0.03$ ). In addition, $>5 \% \mathrm{BWL}$ occurred in 2 patients (8\%) in the EPA-enriched EN group and 10 patients (39\%) in the standard EN group. The authors concluded that perioperative EPA-enriched EN prevented LBL after esophagectomy compared with a standard EN.

However, conflicting findings have been obtained with regard to the LBL. In a separate trial, Healy and Ryan et al. evaluated the clinical effects of EPA-enriched EN in 191 esophageal cancer patients who received esophagectomy (EPA-enriched EN in 97 patients and standard EN in 94 patients) (26). They administered the oral supplementation ProSure ${ }^{\circledR}$ (Abbot Laboratories) at $600 \mathrm{kcal} /$ day (including $2.2 \mathrm{~g} \mathrm{EPA}$ ) from 5 days before surgery to 1 month after esophagectomy. They found that the mean LBL at 1-month post-discharge was $-3.7 \mathrm{~kg}( \pm 8.7 \mathrm{~kg})$ in the standard EN group and $-5.6 \mathrm{~kg}( \pm 12.1 \mathrm{~kg})$ in the EPA-enriched EN group $(p=0.355)$. The percentage of LBL was $6.3 \%$ in the standard EN group and $8.3 \%$ in the EPA-enriched EN group. In addition, the BWL at 1-month post-discharge was $-3.5 \mathrm{~kg}$ $( \pm 3.8 \mathrm{~kg})$ in the standard EN group and $-2.1 \mathrm{~kg}( \pm 11.5 \mathrm{~kg})$ in the EPA-enriched EN group $(p=0.259)$. They concluded that perioperative EPA-enriched EN did not prevent LBL after esophagectomy compared with a standard EN.

\section{Future Perspectives and Ongoing Trials}

Thus far, six randomized clinical trials have evaluated the clinical impact of oral nutritional treatment on perioperative body composition changes in gastrointestinal cancer patients. However, only two have demonstrated the clinical benefits of oral nutritional treatment. This could be the result of the low compliance with oral nutritional treatment. In our study, where 
we used ProSure, the median preoperative compliance with ProSure was $100 \%$, while the median postoperative compliance was 54\% (23). In addition, in Kong's study, almost all patients were able to consume over $250 \mathrm{kcal}$ of ONS daily, but $73.8 \%$ of the patients were unable to tolerate a daily dose of $250 \mathrm{ml}$ of ONS between the day of discharge and the first outpatient clinic visit on the 21st postoperative day (22). The reasons for the low compliance with oral nutritional treatment include changes in the patients' personal preferences after surgery and a reduction in the size of the remnant stomach.

If patients can improve their postoperative oral intake of nutritional treatment, it may be possible to improve the effects of oral nutritional treatment on the body composition change. To this direction, Kobayashi et al. evaluated the clinical effects of ONS on the postoperative BWL in 82 gastric cancer patients who received gastrectomy. They administered Racol ${ }^{\circledR}$ (Otsuka Pharmaceutical Factory) at 400 $\mathrm{kcal} /$ day from 7 days before surgery to 3 months after surgery (27). The primary endpoint of the study was the postoperative percentage weight change at three months after surgery. The median daily oral intake of Racol ${ }^{\circledR}$ was $211 \mathrm{ml}$. They found that the mean weight change after 3 months was 91.7\% among all patients. In addition, when they compared the body weight changes according to compliance with Racol ${ }^{\circledR}$ treatment, a significant difference was noted between the high-adherence group and the low-adherence group (cutoff value: $200 \mathrm{ml}$ ). The mean percentage weight change at 3 months after surgery was $6.1 \%$ in the high-adherence group but $10.4 \%$ in the low-adherence group $(p<0.001)$. Given the above, if the risk factors related to the patient's postoperative oral intake can be identified, it may be possible to select an oral nutritional treatment according to the risk factors.

In addition, to optimize the perioperative oral nutritional treatment for gastric cancer patients, several points need to be clarified. First, the optimal duration and methods of oral nutritional treatment. Second, the clinical benefits of oral nutritional treatment for the long-term oncological outcomes. If these points could be clarified, an effective program might be able to be established for gastrointestinal cancer patients.

Since 2010, several ongoing studies have been examining perioperative oral nutritional treatment for gastrointestinal cancer patients. A Chinese group is conducting a randomized control trial to evaluate the efficacy of an ONS in 374 postoperative gastric cancer patients receiving adjuvant chemotherapy (ClinicalTrials.gov Identifier: NCT03654534). In this trial, they are administering a NUTREN ${ }^{\circledR}$ OPTIMUM (Nestlé Health Science) (400 kcal/400 ml per day) from 7 days after surgery to 3 months after surgery. The primary endpoint will be the postoperative malnutrition and BWL ratio at 1,3 , and 6 months. Another Chinese group is conducting a randomized control trial to evaluate the efficacy of a nutritional education program in 200 patients with gastric cancer (ClinicalTrials.gov Identifier: NCT03952442). The experimental group of patients receives nutrition health education every two weeks and undergoes regular surveys and intervention. The education booklets have been developed based on the guideline (European Society for Clinical Nutrition and Metabolism and American Society for Parenteral and Enteral Nutrition) and characteristics of the patients' disease. The primary endpoint is the risk of malnutrition, body mass index, levels of serum albumin, and quality of life. A Japanese group has also evaluated the clinical impact of an ONS on gastrointestinal cancer patients. Nunobe et al. have evaluated the efficacy of the combination of nutrition and exercise intervention on LBL after surgery for gastric cancer (UMIN000042307). They administered oral supplementation with a single pack of leucine-rich amino acid supplement from day 2 after surgery to 3 months after surgery. Rehabilitation was also performed from day 2 after surgery to 3 months after surgery. The primary endpoint was the change in the percentage LBL at three months after surgery. This trial will terminate in March 2023. Hatao et $a l$. have evaluated the efficacy of super-energy-dense ONSs on the outcomes of postoperative gastric cancer patients (UMIN000041494). They administered super-energy-dense ONSs at approximately $400 \mathrm{kcal} / \mathrm{day}$ and observed subjects from the start of meal intake to 12 weeks after discharge. The primary endpoint was the change in the percentage BWL after surgery. This trial will terminate in March 2022.

\section{Conclusion}

Preoperative body composition changes might have some clinical influence on both the short- and long-term oncological outcomes in gastrointestinal cancer patients. Oral nutritional treatment has proven promising and shown some clinical benefits for gastrointestinal cancer patients during the perioperative period. However, whether or not oral nutritional treatment has clinical benefits on the long-term oncological outcomes in gastrointestinal cancer remains unclear. To optimize the oral nutritional treatment for gastrointestinal cancer patients, it is necessary to clarify the benefits of oral nutritional treatment on the long-term oncological outcomes in gastric cancer patients and establish the optimal approach to administering oral nutritional treatment.

\section{Conflicts of Interest}

The Authors have no conflicts of interest to declare regarding this study.

\section{Authors' Contributions}

Toru Aoyama and Masato Nakazono made substantial contributions to conception and design. Shinnosuke Nagasawa and Kenkis Segami made substantial contributions to acquisition of data, or analysis and interpretation of data. Toru Aoyama and Masato Nakazono have 
been involved in drafting the manuscript or revising it critically for important intellectual content. All Authors have given final approval of the version to be published. Each author should have participated sufficiently in the work to take public responsibility for appropriate portions of the content; and agreed to be accountable for all aspects of the work in ensuring that questions related to the accuracy or integrity of any part of the work are appropriately investigated and resolved. All Authors read and approved the final manuscript.

\section{References}

1 Bray F, Ferlay J, Soerjomataram I, Siegel RL, Torre LA and Jemal A: Global cancer statistics 2018: GLOBOCAN estimates of incidence and mortality worldwide for 36 cancers in 185 countries. CA Cancer J Clin 68(6): 394-424, 2018. PMID: 30207593. DOI: $10.3322 /$ caac. 21492

2 NCCN. NCCN Clinical Practice Guidelines in Oncology. 2018. Available at: http://www.nccn.org [Last accessed on January 15, 2021]

3 Smyth EC, Verheij M, Allum W, Cunningham D, Cervantes A, Arnold D and ESMO Guidelines Committee.: Gastric cancer: ESMO clinical practice guidelines for diagnosis, treatment and follow-up. Ann Oncol 27(suppl 5): v38-v49, 2016. PMID: 27664260. DOI: 10.1093/annonc/mdw350

4 Japanese Gastric Cancer Association: Japanese gastric cancer treatment guidelines 2018 (5th edition). Gastric Cancer 24(1): 121, 2021. PMID: 32060757. DOI: 10.1007/s10120-020-01042-y

5 Argilés G, Tabernero J, Labianca R, Hochhauser D, Salazar R, Iveson T, Laurent-Puig P, Quirke P, Yoshino T, Taieb J, Martinelli E, Arnold D and ESMO Guidelines Committee. Electronic address: clinicalguidelines@esmo.org.: Localised colon cancer: ESMO clinical practice guidelines for diagnosis, treatment and follow-up. Ann Oncol 31(10): 1291-1305, 2020 PMID: 32702383. DOI: 10.1016/j.annonc.2020.06.022

6 Stahl M, Mariette C, Haustermans K, Cervantes A, Arnold D and ESMO guidelines working group.: oesophageal cancer: ESMO clinical practice guidelines for diagnosis, treatment and followup. Ann Oncol 24(Suppl 6): vi51-vi56, 2013. PMID: 24078662. DOI: $10.1093 /$ annonc/mdt342

7 Aoyama T: Perioperative body composition changes in the multimodal treatment of gastrointestinal cancer. Surg Today 50(3): 217-222, 2020. PMID: 31028458. DOI: 10.1007/s00595019-01815-8

8 Yoon SL, Grundmann O, Williams JJ, Gordan L and George TJ Jr: Body composition changes differ by gender in stomach, colorectal, and biliary cancer patients with cachexia: Results from a pilot study. Cancer Med 7(8): 3695-3703, 2018. PMID: 29971962. DOI: 10.1002/cam4.1665

9 Park JY, Park KB, Kwon OK and Yu W: Comparison of laparoscopic proximal gastrectomy with double-tract reconstruction and laparoscopic total gastrectomy in terms of nutritional status or quality of life in early gastric cancer patients. Eur J Surg Oncol 44(12): 1963-1970, 2018. PMID: 30197164. DOI: 10.1016/j.ejso.2018.08.014

10 Matsuura N, Motoori M, Fujitani K, Nishizawa Y, Komatsu H, Miyazaki Y, Miyazaki S, Tomokuni A, Komori T and Iwase K: Correlation between skeletal muscle mass and adverse events of neoadjuvant chemotherapy in patients with gastric cancer. Oncology 98(1): 29-34, 2020. PMID: 31509833. DOI: 10.1159/ 000502613
11 Park HS, Kim HS, Beom SH, Rha SY, Chung HC, Kim JH, Chun YJ, Lee SW, Choe EA, Heo SJ, Noh SH, Hyung WJ, Cheong JH, Kim HI, Son T, Lim JS, Baek SE and Jung M: Marked loss of muscle, visceral fat, or subcutaneous fat after gastrectomy predicts poor survival in advanced gastric cancer: Single-center study from the CLASSIC trial. Ann Surg Oncol 25(11): 3222-3230, 2018. PMID: 30051367. DOI: 10.1245/s10434-018-6624-1

12 Lim HS, Lee B, Cho I and Cho GS: Nutritional and clinical factors affecting weight and fat-free mass loss after gastrectomy in patients with gastric cancer. Nutrients 12(7): 2020. PMID: 32605036. DOI: 10.3390/nu12071905

13 Aoyama T, Yoshikawa T, Shirai J, Hayashi T, Yamada T, Tsuchida K, Hasegawa S, Cho H, Yukawa N, Oshima T, Rino Y, Masuda $M$ and Tsuburaya A: Body weight loss after surgery is an independent risk factor for continuation of S-1 adjuvant chemotherapy for gastric cancer. Ann Surg Oncol 20(6): 20002006, 2013. PMID: 23242818. DOI: 10.1245/s10434-012-2776-6

14 Aoyama T, Kawabe T, Fujikawa H, Hayashi T, Yamada T, Tsuchida K, Yukawa N, Oshima T, Rino Y, Masuda M, Ogata T, Cho $\mathrm{H}$ and Yoshikawa $\mathrm{T}$ : Loss of lean body mass as an independent risk factor for continuation of $\mathrm{s}-1$ adjuvant chemotherapy for gastric cancer. Ann Surg Oncol 22(8): 25602566, 2015. PMID: 25515199. DOI: 10.1245/s 10434-014-4296-Z

15 Aoyama T, Sato T, Maezawa Y, Kano K, Hayashi T, Yamada T, Yukawa N, Oshima T, Rino Y, Masuda M, Ogata T, Cho H and Yoshikawa T: Postoperative weight loss leads to poor survival through poor S-1 efficacy in patients with stage II/III gastric cancer. Int J Clin Oncol 22(3): 476-483, 2017. PMID: 28176023. DOI: $10.1007 / \mathrm{s} 10147-017-1089-y$

16 Aoyama T, Yoshikawa T, Maezawa Y, Kano K, Numata M, Hara K, Komori K, Yamada T, Hayashi T, Sato T, Tamagawa H, Yukawa N, Rino Y, Masuda M, Ogata T, Cho H and Oshima T: The postoperative lean body mass loss at one month leads to a poor survival in patients with locally advanced gastric cancer. J Cancer 10(11): 2450-2456, 2019. PMID: 31258750. DOI: $10.7150 /$ jca.30697

17 Koterazawa Y, Oshikiri T, Takiguchi G, Urakawa N, Hasegawa H, Yamamoto M, Kanaji S, Yamashita K, Matsuda T, Nakamura T, Suzuki S and Kakeji Y: Severe weight loss after minimally invasive oesophagectomy is associated with poor survival in patients with oesophageal cancer at 5 years. BMC Gastroenterol 20(1): 407, 2020. PMID: 33272220. DOI: 10.1186/s12876-02001543-1

18 Mayanagi S, Tsubosa Y, Omae K, Niihara M, Uchida T, Tsushima T, Yokota T, Sato H, Naito T and Yasui H: Negative impact of skeletal muscle wasting after neoadjuvant chemotherapy followed by surgery on survival for patients with thoracic esophageal cancer. Ann Surg Oncol 24(12): 3741-3747, 2017. PMID: 28861809. DOI: 10.1245/s10434-017-6020-2

19 Hatao F, Chen KY, Wu JM, Wang MY, Aikou S, Onoyama H, Shimizu N, Fukatsu K, Seto Y and Lin MT: Randomized controlled clinical trial assessing the effects of oral nutritional supplements in postoperative gastric cancer patients. Langenbecks Arch Surg 402(2): 203-211, 2017. PMID: 27807617. DOI: $10.1007 / \mathrm{s} 00423-016-1527-8$

20 Imamura H, Nishikawa K, Kishi K, Inoue K, Matsuyama J, Akamaru Y, Kimura Y, Tamura S, Kawabata R, Kawada J, Fujiwara Y, Kawase T, Fukui J, Takagi M, Takeno A and Shimokawa T: Effects of an oral elemental nutritional supplement on post-gastrectomy body weight loss in gastric cancer patients: A 
randomized controlled clinical trial. Ann Surg Oncol 23(9): 2928 2935, 2016. PMID: 27084538. DOI: 10.1245/s10434-016-5221-4

21 Kimura Y, Nishikawa K, Kishi K, Inoue K, Matsuyama J, Akamaru Y, Tamura S, Kawada J, Kawase T, Kawabata R, Fujiwara Y, Kanno H, Yamada T, Shimokawa T and Imamura H: Long-term effects of an oral elemental nutritional supplement on post-gastrectomy body weight loss in gastric cancer patients (KSES002). Ann Gastroenterol Surg 3(6): 648-656, 2019. PMID: 31788653. DOI: $10.1002 /$ ags 3.12290

22 Kong SH, Lee HJ, Na JR, Kim WG, Han DS, Park SH, Hong H, Choi Y, Ahn HS, Suh YS and Yang HK: Effect of perioperative oral nutritional supplementation in malnourished patients who undergo gastrectomy: A prospective randomized trial. Surgery 164(6): 12631270, 2018. PMID: 30055788. DOI: 10.1016/j.surg.2018.05.017

23 Ida S, Hiki N, Cho H, Sakamaki K, Ito S, Fujitani K, Takiguchi N, Kawashima Y, Nishikawa K, Sasako M, Aoyama T, Honda M, Sato T, Nunobe $\mathrm{S}$ and Yoshikawa T: Randomized clinical trial comparing standard diet with perioperative oral immunonutrition in total gastrectomy for gastric cancer. Br J Surg 104(4): 377-383, 2017. PMID: 28072447. DOI: 10.1002/bjs.10417

24 Aoyama T, Yoshikawa T, Ida S, Cho H, Sakamaki K, Ito Y, Fujitani K, Takiguchi N, Kawashima Y, Nishikawa K, Oshima T, Nunobe $\mathrm{S}$ and Hiki N: Effects of perioperative Eicosapentaenoic acid-enriched oral nutritional supplement on lean body mass after total gastrectomy for gastric cancer. J Cancer 10(5): 1070-1076, 2019. PMID: 30854113 . DOI: $10.7150 /$ jca.29632
25 Ryan AM, Reynolds JV, Healy L, Byrne M, Moore J, Brannelly N, McHugh A, McCormack D and Flood P: Enteral nutrition enriched with eicosapentaenoic acid (EPA) preserves lean body mass following esophageal cancer surgery: Results of a doubleblinded randomized controlled trial. Ann Surg 249(3): 355-363, 2009. PMID: 19247018. DOI: 10.1097/SLA.0b013e31819a4789

26 Healy LA, Ryan A, Doyle SL, Ní Bhuachalla ÉB, Cushen S, Segurado R, Murphy T, Ravi N, Donohoe CL and Reynolds JV: Does prolonged enteral feeding with supplemental Omega-3 fatty acids impact on recovery post-esophagectomy: Results of a randomized double-blind trial. Ann Surg 266(5): 720-728, 2017. PMID: 28742713. DOI: 10.1097/SLA.0000000000002390

27 Kobayashi D, Ishigure K, Mochizuki Y, Nakayama H, Sakai M, Ito S, Kojima H, Kajikawa M, Ando M and Kodera Y: Multiinstitutional prospective feasibility study to explore tolerability and efficacy of oral nutritional supplements for patients with gastric cancer undergoing gastrectomy (CCOG1301). Gastric Cancer 20(4): 718-727, 2017. PMID: 27885538. DOI: 10.1007/s10120016-0668-3

Received February 15, 2021

Revised February 22, 2021

Accepted February 23, 2021 